\title{
Isolados de Metarhizium anisopliae (Metchnikoff) Sorokin (Hypocreales: Clavicipitaceae) para controle de Diatraea saccharalis Fabricius (Lepidoptera: Crambidae)
}

Eduardo Kenji Hayashida1, Samir Oliveira Kassab² ${ }^{\bowtie}$, Elisângela de Souza Loureiro ${ }^{3}$, Camila Rossoni² Rogério Hidalgo Barbosa², Antônio de Souza Silva² \& Daniele Perassa Costa ${ }^{2}$

1. Sem vínculo institucional, e-mail: ekhayashida@hotmail.com. 2. Universidade Federal da Grande Dourados, e-mail: $\underline{\text { samirkassab@gmail.com }}$ (Autor para correspondência ${ }^{\bowtie}$ ), camilasrossoni@gmail.com, hidalgo.rogerio@gmail.com, antoniobios@yahoo.com.br, daniperassa@gmail.com. 3. Universidade Federal de Mato Grosso do Sul, e-mail: elisangela.loureiro@ufms.br

\section{EntomoBrasilis 7 (1): 20-23 (2014)}

Resumo. A seleção de fungos entomopatogênicos é necessária para que se conheça a eficiência de diferentes isolados e se possa eleger o mais adequado para sua utilização em programas de controle biológico. Este trabalho teve por objetivo avaliar a eficiência dos isolados de Metarhizium anisopliae (Metchnikoff) Sorokin (UFGD 03, UFGD 05, UFGD 07 e UFGD 22), obtidos em Mato Grosso do Sul, em Diatraea saccharalis Fabricius. O ensaio experimental foi composto por um delineamento inteiramente casualizado com 5 tratamentos e 10 repetições, com 5 lagartas padronizadas por tamanho em cada repetição. De maneira geral, $M$. anisopliae afetou o ciclo biológico de $D$. saccharalis e o desenvolvimento desta praga é afetado pelo fungo. A percentagem de lagartas que empuparam variou de 64,00\% a 88,60\%. O tratamento com o isolado UFGD o3 apresentou maior percentagem de pupas mortas (68,00\%) em relação aos demais isolados testados e quando comparado ao valor da testemunha (11,00\%). A emergência dos adultos de $D$. saccharalis foi de $87,02 \%$ na testemunha. Nos tratamentos com o fungo, houve variação de 35,20\%, 38,00\%, 40,00\%, 52,80\% proporcionada pelos isolados UFGD 22, UFGD 03, UFGD 05 e UFGD 07, respectivamente.

Palavras-Chave: Controle biológico; cana-de-açúcar; fungos entomopatogênicos.

\section{Isolates of Metarhizium anisopliae (Metchnikoff) Sorokin (Hypocreales: Clavicipitaceae) for the control of Diatraea saccharalis Fabricius (Lepidoptera: Crambidae)}

Abstract. The selection of entomopathogenic fungus that is a necessary to know the efficiency of different isolates and one can choose the most suitable for use in biological control programs. This study aimed to evaluate the efficiency of the isolates of Metarhizium anisopliae (Metchnikoff) Sorokin (UFGD 03, UFGD 05, UFGD UFGD o7 and UFGD 22), obtained in Mato Grosso do Sul on the Diatraea saccharalis Fabricius. The experimental trial consisted of a completely randomized design with five treatments and 10 repetitions with 5 caterpillars standardized size in each repetition. In general, $M$. anisopliae affects the life cycle of $D$. saccharalis and development of this pest is affected by the fungus. The percentage of caterpillars that pupae ranged from $64.00 \%$ to $88.60 \%$. Treatment with isolated UFGD 03 had a higher percentage of dead pupae (68.00\%) compared to other isolates tested and compared to the control value (11.00\%). The adult emergence of $D$. saccharalis was $87.02 \%$ in the control. In the treatments with the fungus, there was a variation of $35.20 \%, 38.00 \%, 40.00 \%, 52.80 \%$ provided by isolates UFGD 22, UFGD 03, UFGD 05 and UFGD 07, respectively.

Keywords: Biological control; entomopathogenic fungus; sugarcane.

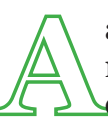
área cultivada com cana-de-açúcar no Brasil é de oito milhões de hectares e na safra 2010/2011, a indústria canavieira, beneficiou 625 milhões de toneladas de cana para a produção de açúcar e etanol (ConAB 2012). O Estado de Mato Grosso do Sul destaca-se como o quinto produtor no ranking nacional e atualmente $571 \mathrm{mil}$ ha são destinados ao cultivo de cana-de-açúcar (CANASAT 2012).

Apesar da importância da cultura, diversos insetos se alimentam da cana-de-açúcar e quando suas populações aumentam de maneira desordenada, que pode ser devido ao favorecimento, por condições climáticas e ausência de inimigos naturais esses causam prejuízos econômicos sendo denominados pragas (SEGATO et al. 2006).

Dentre os problemas fitossanitários encontrados na cultura, destaca-se a Diatraea saccharalis Fabricius (Lepidoptera: Crambidae) que é uma das principais pragas da cana-de-açúcar (DinARDO-MIRANDA et al. 2012). As injúrias causadas pelas fases imaturas, dessa espécie, promovem prejuízos direitos por meio da abertura de galerias no caule e indiretos devido às infecções de microrganismos, resultando em perdas na produção de açúcar e álcool.

A utilização de inseticidas químicos é pouco eficiente no controle da $D$. saccharalis, pois lagartas de $3^{\circ}$ instar habitam galerias no colmo da cana-de-açúcar (PINTO 2009). Assim há necessidade de alternativas biológicas para o controle desta praga e o interesse pela utilização de parasitoides, predadores e entomopatógenos tem aumentado.

Atualmente, o método de controle mais eficiente de D. saccharalis têm sido realizado por meio do endoparasitoide larval Cotesia flavipes Cameron (Hymenoptera: Braconidae) (Silva et al. 2012). Pesquisas também foram desenvolvidas com parasitoide de ovos da espécie Trichogramma galloi Zucchi (Hymenoptera: Trichogrammatidae) (Pereira-Barros et al. 2005). Outra alternativa para o controle de $D$. saccharalis pode ser a utilização dos fungos entomopatogênicos. 
T A broca D. saccharalis é suscetível aos fungos entomopatogênicos e os testes realizados em campo, com a espécie Beauveria bassiana (Balsamo) Vuillemin (Ascomycetes: Clavicipitaceae) causou mortalidade média de $44 \%$ e 89 para as concentrações de $10^{13}$ conídios.ha ${ }^{-1}$ e $5 \times 10^{8}$ conídio.mL $\mathrm{m}^{-1}$, respectivamente (Alves 1998; Wenzel et al. 2006). Em outros trabalhos, o uso de suspensões fúngicas de B. bassiana e Metarhizium anisopliae (Metchnikoff) Sorokin (Hypocreales: Clavicipitaceae), em doses subletais, comprometeu as características biológicas de $D$. saccharalis, tais como longevidade, fecundidade e viabilidade de ovos (Oliveira et al. 2008).

Dessa forma, é primordial o incremento e aperfeiçoamento de pesquisas direcionadas a avaliação do potencial e seleção dos fungos entomopatogênicos para que se conheça a eficiência de diferentes isolados e se possa eleger o mais adequado no combate a praga e implementar a sua utilização em programas de controle biológico da D. saccharalis.

Este trabalho objetivou avaliar a eficiência dos isolados de $M$. anisopliae (UFGD 03, UFGD 05, UFGD 07 e UFGD 22), obtidos em Mato Grosso do Sul, Brasil sobre lagartas de D. saccharalis.

\section{MATERIAL E MÉTODOS}

O experimento foi conduzido no laboratório de Microbiologia da Faculdade de Ciências Biológicas e Ambientais da Universidade Federal da Grande Dourados (UFGD), em Dourados, Mato Grosso do Sul, com as etapas:

Criação de $D$. saccharalis. As lagartas utilizadas na pesquisa foram provenientes da criação do laboratório Biossoluções, município de Dourados (MS). Criadas seguindo a metodologia adaptada de PARRA (2007), onde ovos de D. saccharalis foram colocados em frascos de vidro $(8,5 \mathrm{~cm}$ de diâmetro e $13 \mathrm{~cm}$ de altura) contendo dieta artificial para alimentação das lagartas recém eclodidas, onde permaneceram até o último instar. Essas lagartas foram transferidas para placas de Petri descartáveis $(6,5$ $\mathrm{cm}$ de diâmetro e 2,5 cm de altura) com dieta de realimentação até a formação das pupas. As pupas foram recolhidas, selecionadas por características morfológicas e acondicionadas em potes plásticos telados, permanecendo até a fase adulta. Os adultos foram separados em casais com 20 machos e 30 fêmeas, e colocados em gaiolas de tubos de PVC (10 cm de diâmetro e 22 de altura). As gaiolas foram fechadas com papel sulfite e elástico, revestidas internamente com folhas de papel sulfite como substrato para oviposição sendo os ovos coletados diariamente, lavados e armazenados em câmara climatiza à temperatura de 25 $\pm 2^{\circ} \mathrm{C}$, umidade relativa de $70 \pm 10 \%$ umidade relativa (U.R.) e fotofase de $14 \mathrm{~h}$ para a continuidade da criação.

Obtenção e produção de $M$. anisopliae. Os isolados de $M$. anisopliae utilizados na pesquisa foram provenientes da Coleção de Microrganismos Entomopatogênicos do laboratório de Microbiologia da Faculdade de Ciências Biológicas e Ambientais (FCBA), da Universidade Federal da Grande Dourados (UFGD) (Tabela 1).

Tabela 1. Isolados de Metarhizium anisopliae (Metchnikoff) Sorokin (Hypocreales: Clavicipitaceae) utilizados no experimento.

\begin{tabular}{|ccll}
\hline Espécie & Isolado & Hospedeiro & Procedência \\
\hline & UFGD 03 & & \\
$\begin{array}{c}\text { Metarhizium } \\
\text { anisopliae }\end{array}$ & UFGD 05 & $\begin{array}{l}\text { Mahanarva } \\
\text { fimbriolata } \\
\text { (Hemiptera: } \\
\text { Cercopidae) }\end{array}$ & $\begin{array}{l}\text { Dourados, Mato } \\
\text { Grosso do Sul }\end{array}$ \\
\hline & UFGD 07 & & \\
\hline
\end{tabular}

Os fungos entomopatogênicos foram repicados em meio de cultura BDA (batata-dextrose-ágar) e incubados em câmara climatizada tipo BOD a $25 \pm 2^{\circ} \mathrm{C}$ e fotofase de $12 \mathrm{~h}$ e $70 \pm 10 \%$ (U.R) (Alves 1998). Ressalta-se ainda que os fungos entomopatogênicos utilizados nesta pesquisa apresentaram germinação dos conídios acima de $97 \%$ no momento da realização dos bioensaios.

Desenvolvimento Experimental. As suspensões fúngicas utilizadas no bioensaio foram obtidas a partir dos isolados, raspando-se a superfície do meio de cultura com o auxilio de uma alça de níquel-cromo. Os conídios do fungo $M$. anisopliae foram transferidos para tubos de ensaio contendo $10 \mathrm{~mL}$ de água destilada esterilizada e $0,1 \mathrm{~mL}$ de Tween $80^{\circledR}$ a $0,1 \%$. Para a montagem do experimento foi preparada uma suspensão com concentração de $10^{9}$ conídios. $\mathrm{mL}^{-1}$ quantificada em câmara de Neubauer ${ }^{\circledR}$ com o auxílio de um microscópio óptico (Alves 1998).

O ensaio experimental foi composto por um delineamento inteiramente casualizado com cinco tratamentos e 10 repetições. Cada repetição composta por cinco lagartas de D. saccharalis padronizadas por tamanho em cada repetição. Para a infecção do patógeno, cada lagarta foi tratada com 0,5 mL de suspensão fúngica (inoculação tópica). Cada placa de Petri descartável (6 cm de diâmetro) contendo um quadrado $(4 \mathrm{~cm} \mathrm{x} 4 \mathrm{~cm})$ de dieta artificial recebeu cinco lagartas de $D$. saccharalis, perfazendo um total de 10 placas (repetição) por tratamento, ou seja, 50 insetos. Posteriormente os tratamentos foram acondicionados em sala climatizada à temperatura de $25 \pm 2^{\circ} \mathrm{C} \mathrm{e} 70 \pm 10 \%$ (U.R) e fotoperíodo de $12 \mathrm{~h}$.

As avaliações foram realizadas no intervalo de três dias por um período de 15 dias. Cada inseto morto foi transferido para novas placas de Petri com algodão hidrófilo levemente umedecido com água destilada esterilizada, acondicionadas em BOD $\left(25 \pm 2^{\circ}\right.$ C e $70 \pm 10 \%$ (U.R) e fotoperíodo de $12 \mathrm{~h}$ ) para verificação do crescimento do patógeno.

As características avaliadas foram: mortalidade de lagartas e pupas, pupação e emergência de adultos de $D$. saccharalis. Os dados obtidos nos tratamentos foram calculados com a fórmula de Аввотт (1925) e submetidos à análise de variância (teste F) e quando significativos comparados pelo teste de Tukey a $5 \%$ de probabilidade.

\section{RESULTADOS E DISCUSSÃO}

De maneira geral, $M$. anisopliae afeta o ciclo biológico de $D$. saccharalis. Isto é importante, pois, a interferência dos fungos entomopatogênicos nos diferentes estádios desta praga pode auxiliar no manejo fitossanitário de $D$. saccharalis (Figura 1). A eficiência dos isolados UFGD 03, UFGD 05, UFGD o7 e UFGD 22 sobre lagartas de $D$. saccharalis foi de 36,00\%, 30,80\%, 17,60\% e $21,80 \%$ respectivamente (Tabela 2 ).

A porcentagem de lagartas ao estádio de pupas variou de 64,00\% a 88,60\%. O isolado UFGD o7 apresentou a maior porcentagem de pupas viáveis $(82,40 \%)$. O tratamento com o isolado UFGD o3 apresentou maior porcentagem de pupas mortas $(68,00 \%)$ em relação aos demais isolados testados e quando comparado ao valor da testemunha (11,00\%) (Tabela 2). A emergência dos adultos de $D$. saccharalis foi de $87,02 \%$ na testemunha. Nos tratamentos com o fungo, houve variação de $35,20 \%, 38,00 \%$, $40,00 \%, 52,80 \%$ proporcionada pelos isolados UFGD 22, UFGD 03, UFGD 05 e UFGD 07, respectivamente (Tabela 2).

Dezoito lagartas de $D$. saccharalis foram mortas no tratamento com o isolado 03. Nos demais tratamentos o número de lagartas mortas foi de 15,4; 10,9 e 8,8 para os isolados UFGD 05, UFGD 22 e UFGD 07, respectivamente. A porcentagem de pupas com emergência da praga para os isolados UGFD 03, UFGD22, UFGD 05, UFGD 07, foi de 12,16\%, 13,76\%, 13, 84\% e 21,75, respectivamente. 


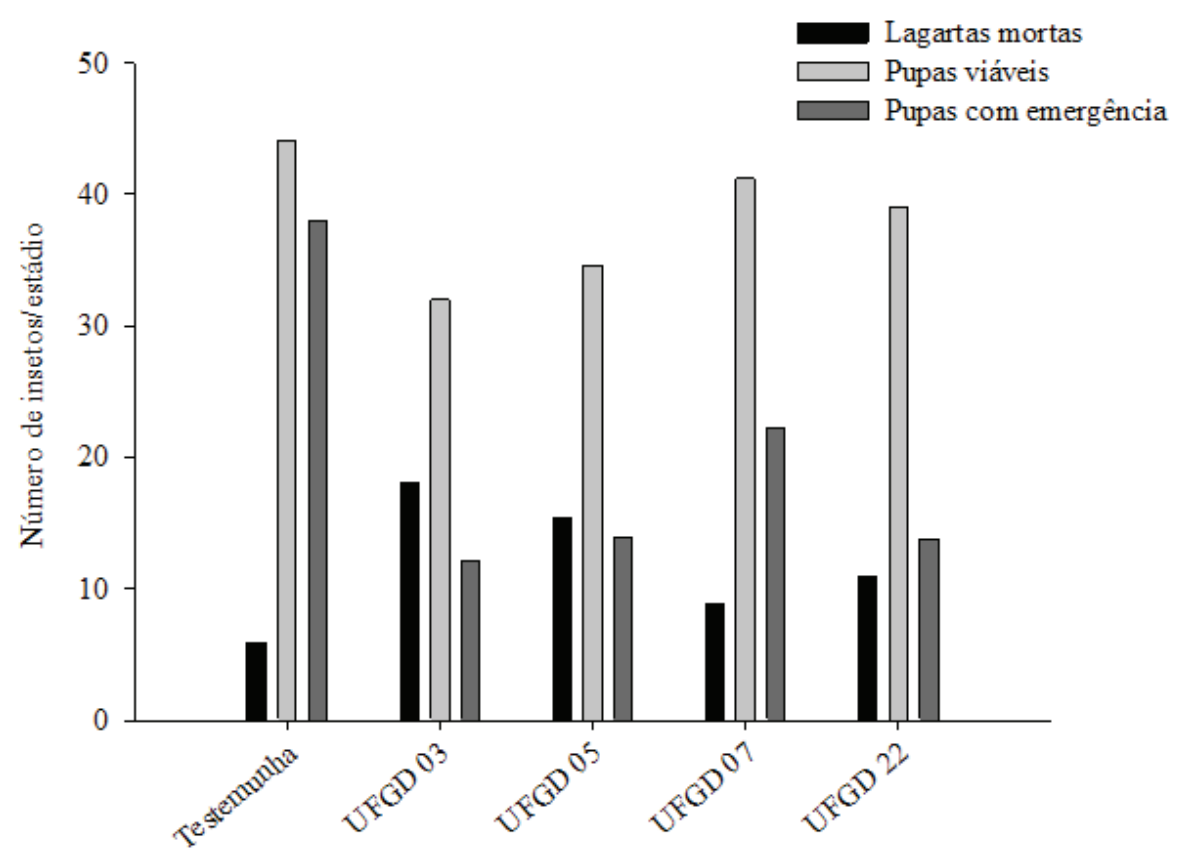

Figura 1. Lagartas mortas, pupas viáveis e com emergência de adultos de Diatraea saccharalis Fabricius (Lepidoptera: Crambidae) em função dos tratamentos com Metarhizium anisopliae (Metchnikoff) Sorokin (Hypocreales: Clavicipitaceae). Temperatura $25 \pm 2^{\circ} \mathrm{C}$, fotofase de $12 \mathrm{~h}$ e $70 \pm 10 \%$ de umidade relativa, mantidas constantes. Dourados-MS, 2012.

Tabela 2. Metarhizium anisopliae (Metchnikoff) Sorokin (Hypocreales: Clavicipitaceae) sobre o desenvolvimento (lagarta-adulto) de Diatraea saccharalis Fabricius (Lepidoptera: Crambidae). Temperatura $25 \pm 2^{\circ} \mathrm{C}$, fotofase de $12 \mathrm{~h}$ e $70 \pm 10 \%$ de umidade relativa, mantidas constantes. Dourados, MS, 2012.

\begin{tabular}{|c|c|c|c|c|}
\hline \multirow[b]{2}{*}{ Tratamentos } & \multicolumn{4}{|c|}{ Características avaliadas } \\
\hline & Mortalidade de lagartas (\%) & Pupação (\%) & $\begin{array}{l}\text { Mortalidade de } \\
\text { pupas (\%) }\end{array}$ & $\begin{array}{c}\text { Emergência de } \\
\text { adultos (\%) }\end{array}$ \\
\hline Testemunha (não tratada) & $11,80 \pm 0,26 \mathrm{~b}$ & $88,60 \pm 0,10$ a & $11,00 \pm 0,30 \mathrm{~b}$ & $87,02 \pm 0,43$ a \\
\hline UFGD o3 ( $1 \times 10^{9}$ con.mL $\left.L^{-1}\right)$ & $36,00 \pm 0,76 \mathrm{a}$ & $64,00 \pm 0,57 \mathrm{~b}$ & $68,00 \pm 1,04$ a & $38,00 \pm 0,75 \mathrm{~b}$ \\
\hline UFGD o5 ( $1 \times 10^{9}$ con.mL $\left.L^{-1}\right)$ & $30,80 \pm 1,32 \mathrm{ab}$ & $69,20 \pm 0,88 \mathrm{ab}$ & $66,40 \pm 0,66 \mathrm{a}$ & $40,00 \pm 0,70 \mathrm{~b}$ \\
\hline UFGD $07\left(1 \times 10^{9}\right.$ con.mL $\left.L^{-1}\right)$ & $17,60 \pm 1,18 \mathrm{ab}$ & $82,40 \pm 0,54 a b$ & $61,00 \pm 0,71 \mathrm{a}$ & $52,80 \pm 0,58 b$ \\
\hline UFGD $22\left(1 \times 10^{9}\right.$ con.mL $\left.L^{-1}\right)$ & $21,80 \pm 0,83 a b$ & $78,20 \pm 0,44 a b$ & $62,80 \pm 0,84 \mathrm{a}$ & $35,20 \pm 0,66 \mathrm{~b}$ \\
\hline CV (\%) & 45,5 & 13,8 & 24,92 & 27,11 \\
\hline
\end{tabular}

Médias seguidas de mesma letra não diferem entre si pelo teste de Tukey ao nível de $5 \%$ de probabilidade.

Conídios. $\mathrm{mL}^{-1}=$ con. $\mathrm{mL}^{-1}$

$M$. anisopliae interfere no ciclo biológico de $D$. saccharalis e o desenvolvimento biológico desta praga é afetado pelo fungo. Pode-se constatar que a infecção com as os isolados foi capaz de alterar parte do ciclo de vida (lagarta-adulto) da broca da canade-açúcar.

O efeito obtido pelos isolados de M. anisopliae (UFGD 03, UFGD 05, UFGD 07, UFGD 22) pode ser considerado promissor. Este fato é importante, pois o modo de ação dos fungos entomopatogênicos difere dos inseticidas. Os fungos entomopatogênicos apresentam capacidade de aumento da densidade do patógeno pela transmissão horizontal dos conídios na cultura e há repetições do ciclo da doença na população hospedeira (praga) (HAJEK \& ST. LEGER 1994). Este aumento da doença controla naturalmente a ressurgência da praga e diminui as infestações das populações dos insetos prejudiciais na cultura.

Os isolados na concentração de $10^{9}$ causaram mortalidade de lagartas e pupas de $D$. saccharalis próxima a obtida nos estudos conduzidos com o isolado IBCB 66 (B. bassiana) que proporcionou eficiência de 89,00\% na concentração de $5 \times 10^{8}$ em condições de campo (WeNzel et al. 2006). Isto é importante, pois, alguns autores consideram que os entomopatógenos são eficazes quando apresentam valores de mortalidade acima de 40\% para as pragas estudadas (Alves et al. 1998).
Os fungos B. bassiana e $M$. anisopliae apresentaram atividade patogênica a espécie $D$. saccharalis em condições de campo e laboratório, sendo sugerida a utilização deste entomopatógeno em cana-de-açúcar, contribuindo com o controle desta praga em ambientes com ocorrência natural do inseto (DEstéfANo et al. 2004; Oliveira et al. 2008). Nos estudos, os autores concluíram nos tratamentos com fungo houve redução do número de danos produzidos pelo lepidóptero praga.

Ressalta-se ainda que as condições climáticas de Mato Grosso do Sul são propícias ao desenvolvimento dos fungos entomopatogênicos e isto aumenta possibilidade de controle desta praga. Neste contexto, nos tratamentos com a espécie $M$. anisopliae observa-se que os isolados podem ser utilizados para o controle de $D$. saccharalis sendo o isolado UFGD 03 o mais indicado para contribuir com o Manejo integrado da broca da cana-de-açúcar para as condições do Estado de Mato Grosso do Sul, Brasil.

\section{REFERÊNCIAS}

Abbott, W.S.A., 1925. A method of computing the effectiveness of an inseticide. Journal of Economic Entomology, 18: 265267.

Alves, S.B., 1998. Fungos entomopatogênicos, p. 289-381. In: 
Alves, S.B. (Ed). Controle microbiano de insetos. Piracicaba: FEALQ, Piracicaba, $1163 \mathrm{p}$.

Canasat - Monitoramento da cana-de-açúcar via imagens de satélite, 2012. Tabelas. Disponível em: <http://www.dsr.inpe. $\mathrm{br} / \mathrm{laf} /$ canasat/tabelas.html $>$ [data de acesso 10/07/2012].

Conab - Companhia Nacional de Abastecimento, 2012. Dados sobre projeção e consumo da cana-de-açúcar. Disponível em: $<$ http://www.conab.gov.br> [data de acesso 11/07/2012].

Destéfano, R.H.R., S.A.L. Destéfano \& C.L. Messias, 2004. Detection of Metarhizium anisopliae var. anisopliae within infected sugarcane borer Diatraea saccharalis (Lepidoptera, Pyralidae) using specific primers. Genetics and Molecular Biology, 27: 245-252.

Dinardo-Miranda, L.L., I.A. Anjos, V.P. Costa \& J.V. Fracasso, 2012. Resistance of sugarcane cultivars to Diatraea saccharalis. Pesquisa Agropecuária Brasileira, 47: 1-7.

Hajek, A.E. \& R.J.S.T. Leger, 1994. Interactions between fungal pathogens and insect hosts. Annual Review of Entomology, 39: 293-322.

Oliveira, M.A.P. de, E.J.Marques, V.W.Teixeira \& R. Barros, 2008. Efeito de Beauveria bassiana (Bals.) Vuill. e Metarhizium anisopliae (Metsch.) Sorok. sobre características biológicas de Diatraea saccharalis F. (Lepidoptera: Crambidae). Acta Scientiarum: Agronomy, 30: 220-224.

Parra, J.R.P., 2007. Técnicas de criação de insetos para programa de controle biológico. $6^{\mathrm{a}}$ ed. Piracicaba, FEALQ, 134 p.
Pereira-Barros, J.L., S.M.F. Broglio-Micheletti, A.J.N. Santos, L.W.T. Carvalho, L.H.T. Carvalho \& C.J.T. Oliveira, 2005. Aspectos biológicos de Trichogramma galloi Zucchi, 1988 (Hymenoptera: Trichogrammatidae) criados em ovos de Diatraea saccharalis (Fabricius, 1794) (Lepidoptera: Crambidae). Ciência e Agrotecnologia, 29: 714-718.

Pinto, A.S., 2009. Guia ilustrado de pragas da cana-de-açúcar. Piracicaba, Piracicaba: CP2, 160 p.

Segato, S.V., A.S. Pinto, E. Jendiroba \& J.C.M. Nóbrega, 2006. Atualização em produção de cana-de-açúcar. Piracicaba, Piracicaba: CP2, 415 p.

Silva, C.C.M., E.J. Marques, J.V. Oliveira \& E.C.N. Valente, 2012. Preference of the parasitoid Cotesia flavipes (Cam.) (Hymenoptera: Braconidae) for Diatraea (Lepidoptera: Crambidae). Acta Scientiarum: Agronomy, 34: 23-27.

Wenzel, I.M., F.H.C. Giometti \& J.E.M. Almeida, 2006. Patogenicidade do isolado IBCB 66 de Beauveria bassiana à broca da cana-de-açúcar Diatraea saccharalis em condições de laboratório. Arquivos do Instituto Biológico, 73: 259-261.

\section{Recebido em: 05/04/2013}

Aceito em: 3o/o9/2013

\section{Como citar este artigo:}

Hayashida, E.K., S.O. Kassab, E.S. Loureiro, C. Rossoni, R.H. Barbosa, A.S. Silva \& D.P. Costa, 2014. Isolados de Metarhizium anisopliae (Metchnikoff) Sorokin (Hypocreales: Clavicipitaceae) para Controle de Diatraea saccharalis Fabricius (Lepidoptera: Crambidae), 7 (1): $20-23$. Acessível em: doi:10.12741/ebrasilis.v7i1.323
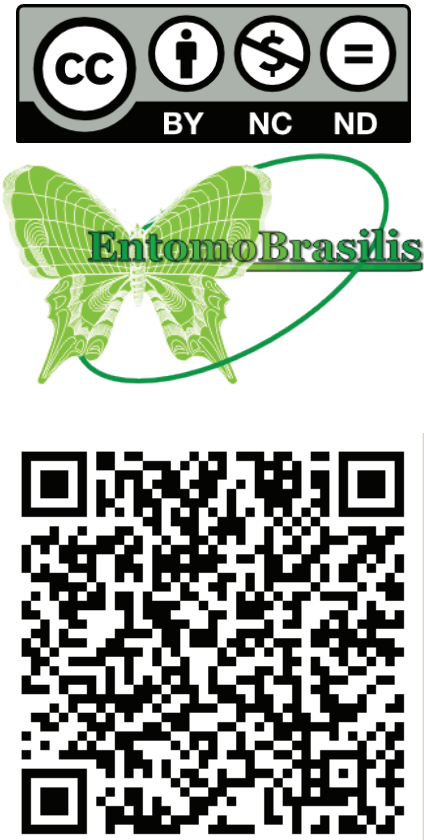